\title{
SITUACIÓN DE LOS RECURSOS HUMANOS DE ACTIVIDAD FÍSICA Y DEPORTE EN LOS AYUNTAMIENTOS DE LA COMUNIDAD VALENCIANA (ESPAÑA)
}

\author{
STATUS OF HUMAN RESOURCES FOR VALENCIAN COMMUNITY (SPAIN) REGARDING \\ PHYSICAL ACTIVITY AND SPORT
}

\author{
Gustavo Martínez Serrano ${ }^{1}$ \\ Antonio Campos Izquierdo ${ }^{2}$ \\ Carlos Pablos Abella ${ }^{3}$ \\ Juan Antonio Mestre Sancho ${ }^{4}$
}

Resumen

Los recursos humanos de la actividad física y del deporte de los ayuntamientos de la Comunidad Valenciana, tienen como una de sus finalidades el fomento de la actividad físico-deportiva en el municipio correspondiente, a través de la elaboración y ejecución de actividades y programas de promoción del deporte, dirigidos a todos los sectores de la población y prestando una atención especial al deporte escolar, a las personas discapacitadas y a las personas mayores, por lo que representa un campo de interés científico y social que requiere ser investigado. Mediante una metodología cuantitativa de corte descriptivo y el empleo de la entrevista estandarizada como instrumento de recogida de información, se reproducen aquellas características más relevantes observadas en la Comunidad Valenciana. Los resultados describen que las personas que trabajan en funciones físico-deportivas en los ayuntamientos son mayoritariamente hombres, que desarrollan una amplia variedad de funciones, fundamentalmente docencia de actividad física y/o deporte y tareas de organización y coordinación, y en situaciones laborales dispares. Asimismo, el estudio revela que la gran mayoría desea continuar en su trabajo actual, habiendo conseguido su puesto laboral a través de su biografía personal o curriculum vitae y teniendo fundamentalmente titulación deportiva de entrenador o monitor.

Palabras clave: recursos humanos, ayuntamientos, deporte, actividad física, gestión deportiva.

Abstract

Human resources for Valencian Community physical activity and sport have a critical aim to support them in some relevant populations through a development and implementation of activities and programs to promote sports. It is led to every segment of population to pay special attention to school sports, disabled and elderly, representing therefore a scientific and social interest field that requires to be explored. Using a quantitative and descriptive methodology and standardized interviews as data collection instrument, we replicate those most relevant features seen in Valencia. Results describe

1 Doctor y licenciado en Ciencias de la Actividad Física y del Deporte. Diplomado Magisterio especialidad Educación Física. Generalitat Valenciana - Conselleria de Educación, Formación y Empleo. Correo electrónico: gusmarse@alumni.uv.es

2 Doctor y licenciado en Ciencias de la Actividad Física y del Deporte. Profesor FCCAFD-INEF Madrid, Universidad Politécnica de Madrid, Facultad de Ciencias de la Actividad Física y del DeporteINEF. Correo electrónico: antoniociz@yahoo.es

3 Doctor en Psicología y licenciado en Educación Física. Profesor FCCAFD UCV, centro de trabajo: Universidad Católica de Valencia San Vicente Mártir, Facultad de Ciencias de la Actividad Física y del Deporte. Correo electrónico: Carlos.pablos@ucv.es

4 Doctor en Psicología y licenciado en Educación Física. Profesor FCCAFD UCV, Universidad Católica de Valencia San Vicente Mártir, Facultad de Ciencias de la Actividad Física y del Deporte. Correo electrónico: jamestre@ucv.es 
Situación de los recursos humanos de actividad física y deporte en los ayuntamientos de la

comunidad valenciana (España). Gustavo Martínez Serrano, Antonio Campos Izquierdo, Carlos

Pablo Abella y Juan Antonio Mestre Sancho

people who perform physical and sports roles at municipalities, but mostly are men who develop a wide variety of jobs, primarily teaching physical activity and/or sport and organization and coordination tasks, but dissimilar job conditions are relevant. This analysis also reveals that vast majority of them want to continue performing their current job, achieved through a personal biography or a resume basically as sport coach or a teaching assistant.

Keywords: Human resources, municipalities, sport, physical activity, sport management.

Fecha de recepción: 26 de abril de 2012

Fecha de aprobación: 12 de septiembre de 2012

\section{Marco teórico}

García Ferrando (2006) explica que los ciudadanos reconocen cada vez más a los ayuntamientos como los entes de la administración pública a los cuales dirigirse para llevar a cabo gestiones relacionadas con el deporte. Según este autor, los Servicios Deportivos Municipales han adquirido, con el avance del Estado de las Autonomías, más responsabilidades, lo que muestra un $61 \%$ de encuestados que, en su estudio sobre los hábitos deportivos de los españoles, señalan el ayuntamiento como la entidad a la cual dirigirse para realizar una gestión relacionada con el servicio deportivo en cada comunidad. Del mismo modo, García Ferrando, Llopis y Mestre (2007) exponen que el Ayuntamiento de Valencia, a través de la Fundación Deportiva Municipal, es la administración pública percibida por los valencianos como más cercana en materia de deporte, lo que implica que esta opinión positiva se ve incrementada a nivel porcentual en relación a los datos obtenidos en el año 2000, cuando se describía un 49\%, hasta situarse en 2006 en el 62\%, ligeramente superior a la media obtenida en toda España (59\%).

Según estos autores, en cuanto a la utilización individual y familiar de los servicios y actividades deportivas que ofrece el Ayuntamiento de Valencia a través de la Fundación Deportiva Municipal, el 35\% de los valencianos manifiesta que los utilizan en la actualidad, frente al $28 \%$ de 2000; el 33\% los ha utilizado anteriormente, pero ahora no (el $24 \%$ en 2000 ), y otro $32 \%$ nunca los ha utilizado, porcentaje que desciende en relación con el año 2000 en el que se situaba próximo del $50 \%$, concretamente en el $47 \%$. De esta manera, la media de utilización de los servicios y actividades deportivas valencianos se sitúa ligeramente por encima de la media nacional, que se encuentra en el $32 \%$, con lo que se observa un amplio margen de utilización de las instalaciones existentes y de los servicios que se ofrecen en ellas en comparación con el resto de regiones de España.

Por consiguiente, el estudio de la situación actual de las personas que trabajan en funciones de actividad física y deporte en los ayuntamientos de la Comunidad Valenciana proyectará una información de relevancia sobre la situación actual de los recursos humanos de esta entidad pública.

\section{Método}

Siguiendo las aportaciones de Campos, Pablos y Mestre (2006a, 2006b), la metodología utilizada es cuantitativa de corte descriptivo y de corte transversal, establecida para dos momentos temporales (épocas de verano y de invierno), siendo los procedimientos utilizados los propios de la encuesta.

El nivel de confianza que se estableció fue de entre +2 sigmas como valores de la distribución normal y donde la probabilidad comprendida en este intervalo es del $95,5 \%$ y el margen de error establecido para la muestra fue de $+4 \%$.

Se realizaron entrevistas individuales y orales a 600 personas que trabajaban en funciones de actividad física y deporte en entidades o instalaciones deportivas de la Comunidad Valenciana (Tabla 1). La entrevista estandarizada por medio de cuestionario de esta investigación, es la utilizada y validada por el profesor Antonio Campos en su estudio sobre la situación profesional de las personas que trabajan en funciones de actividad física y deporte en la Comunidad Valenciana en el año 2005. 
Tabla 1. Distribución de la cantidad de personas entrevistadas y el porcentaje en cada uno de los estratos de población determinados

\begin{tabular}{||c|c|c|c|c|c|c|c|c||}
\hline & \multicolumn{2}{|c|}{ Alicante } & \multicolumn{2}{c|}{ Castellón } & \multicolumn{2}{c|}{ Valencia } & \multicolumn{2}{|c|}{ Total } \\
\hline & $\%$ & Cantidad & $\%$ & Cantidad & $\%$ & Cantidad & $\%$ & Cantidad \\
\hline Más de 100 000 habitantes & 12,7 & 76 & 4 & 24 & 18,7 & 112 & 35,4 & 212 \\
\hline $\begin{array}{c}\text { Entre } 50000 \text { habitantes y } 100 \\
000 \text { habitantes }\end{array}$ & 8 & 48 & 1,3 & 8 & 6 & 36 & 15,3 & 92 \\
\hline $\begin{array}{c}\text { Entre } 20000 \text { habitantes y } 49 \\
999 \text { habitantes }\end{array}$ & 9 & 54 & 3,3 & 20 & 13,3 & 80 & 25,6 & 154 \\
\hline $\begin{array}{c}\text { Entre } 10000 \text { habitantes y } 19 \\
999 \text { habitantes }\end{array}$ & 5,7 & 34 & 1,3 & 8 & 7,7 & 46 & 14,7 & $\mathbf{8 8}$ \\
\hline $\begin{array}{c}\text { Entre } 5000 \text { habitantes y } 9999 \\
\text { habitantes }\end{array}$ & 3,3 & 20 & 1,3 & 8 & 4,4 & 26 & 9 & $\mathbf{5 4}$ \\
\hline Total & 38,7 & 232 & 11,2 & $\mathbf{6 8}$ & 50,1 & 300 & 100 & $\mathbf{6 0 0}$ \\
\hline \hline
\end{tabular}

El tipo de muestreo ha sido aleatorio por conglomerados, dentro del cual la investigación llevada a cabo es un muestreo polietápico, estratificado en primera fase por conglomerados.

\section{Resultados y discusión}

Según Martínez, Campos, Pablos y Mestre (2008a, 2008b), en los ayuntamientos de la Comunidad Valenciana (España) las personas llevan a cabo funciones de actividad física y deporte muy repartidas, pero es la docencia de actividad física y/o deporte, con un 20,4\%, la que destaca en comparación con el resto de funciones. En un siguiente grupo se encuentran las funciones de cuidado y mantenimiento de la forma física en grupos $(16,2 \%)$ y las tareas organizativas y coordinativas de actividad física y deporte (19,2\%) (véase Gráfico 3.1).

Gráfico 3.1. Porcentajes de funciones de actividad física y deporte realizadas en la actualidad

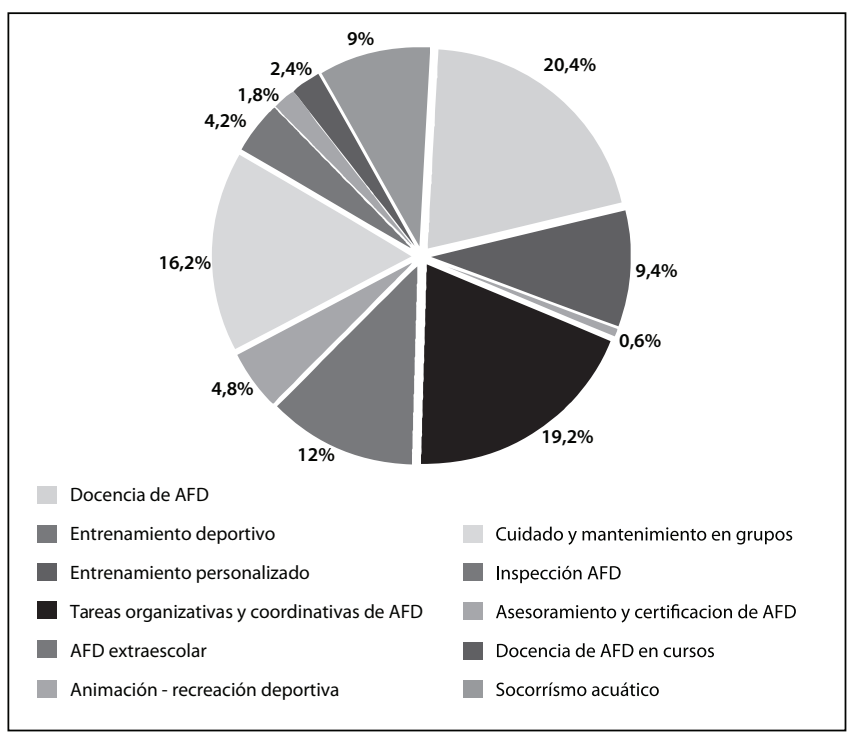

Asimismo, estos autores explican que la función de socorrismo acuático predomina en la época de verano, mientras que las actividades físico-deportivas extraescolares predominan en invierno. La polifuncionalidad de los trabajadores es elevada $(83,4 \%)$, un $70,6 \%$ de ellos ha realizado funciones de actividad física y deporte diferentes a las que desarrolla en la actualidad, la mayoría desenvuelve tareas no relacionadas con la actividad física y el deporte, y un $40 \%$ desea trabajar en funciones de docencia de educación física y/o deportes y tareas organizativas y coordinativas, funciones físico-deportivas más estables y con mejores condiciones laborales (Martínez et al., 2008a).

La docencia sobre contenidos de actividad física y deporte se realiza en cursos subvencionados, reconocidos y/o avalados por entidades públicas, y las tareas más desarrolladas en el área organizativa son las que se refieren a la coordinación y dirección técnica (véase Gráfico 3.2). En esta línea, cabe destacar que un $85 \%$ de estas personas coordina y dirige la labor de otras y un 37,5\% coordina fundamentalmente áreas específicas (Martínez et al., 2008b).

Gráfico 3.2. Tareas en la función de organización y coordinación de actividad física y deporte

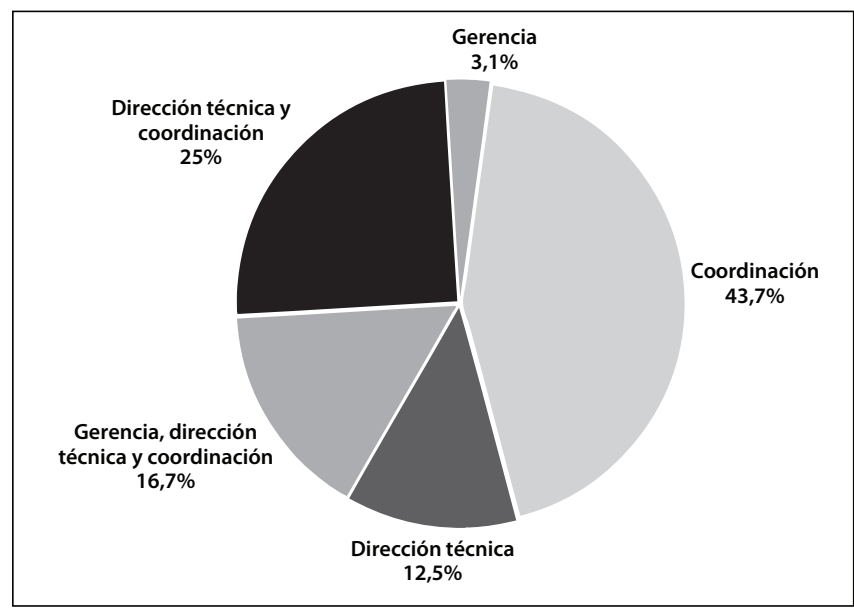

Siguiendo la línea de estos autores, las personas trabajan primordialmente con ciudadanos de la tercera edad y con discapacitados físicos y/o psíquicos, aunque también llevan a cabo labores con embarazadas y/o bebés, con personas con disfunciones sociales y con turistas (véase Gráfico 3.3). Las instalaciones deportivas donde se trabaja son diversas, siendo las más destacadas las instalaciones multideportivas, las instalaciones acuáticas y los centros de enseñanza públicos. 
Gráfico 3.3. Distribución de poblaciones con las que desarrollan funciones de actividad física y deporte

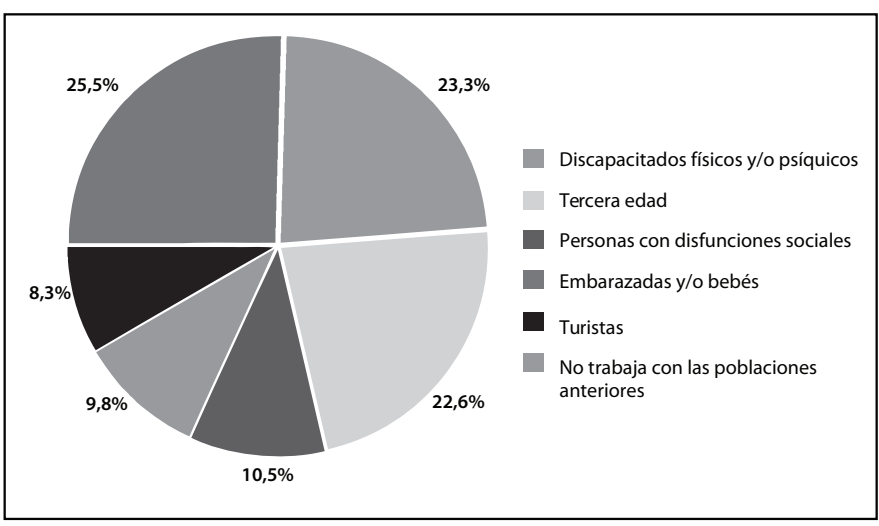

Con respecto a las características sociodemográficas, un $41,2 \%$ de las personas entrevistadas son mujeres y un $58,8 \%$ son hombres. La edad media se sitúa en 33,96 años, lo que determina que los ayuntamientos y la Generalitat Valenciana representan las entidades físico-deportivas con mayor madurez de sus trabajadores, siendo las funciones más longevas la inspección, el asesoramiento y certificación sobre actividad física y deporte y las tareas de organización y coordinación deportiva (Martínez et al., 2008b). El origen se sitúa esencialmente en la Comunidad Valenciana (España).

Gráfico 3.4. Género de las personas que trabajan para los ayuntamientos

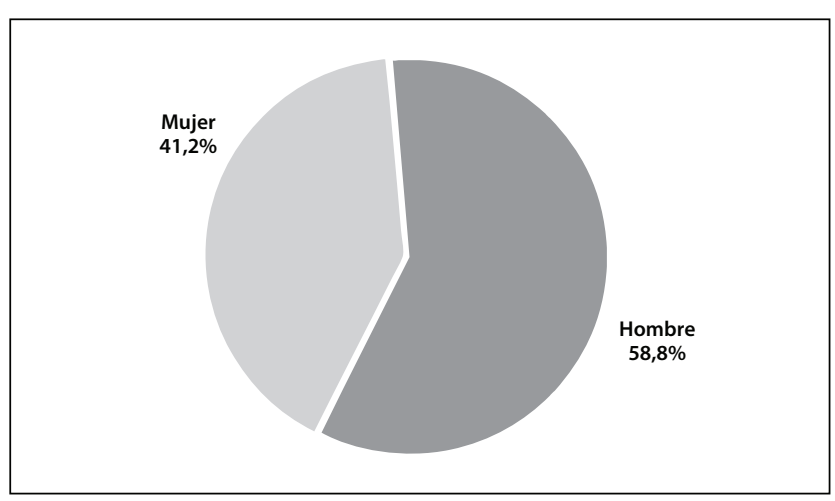

Un $23,9 \%$ de las relaciones laborales representa situaciones sin contratación, el pluriempleo representa un $33,2 \%$ y se describe un equilibrio entre los contratos temporales por obra o servicio y los contratos indefinidos del tipo funcionario o laboral (Martínez, 2009). Este equilibrio evidencia una dualidad laboral, superando las situaciones temporales el porcentaje de temporalidad $(37,4 \%)$ establecido de media en la Comunidad Valenciana (Instituto Valenciano de Estadística [IVE], 2006a, 2006b).
Gráfico 3.5. Distribución de los tipos de relaciones laborales

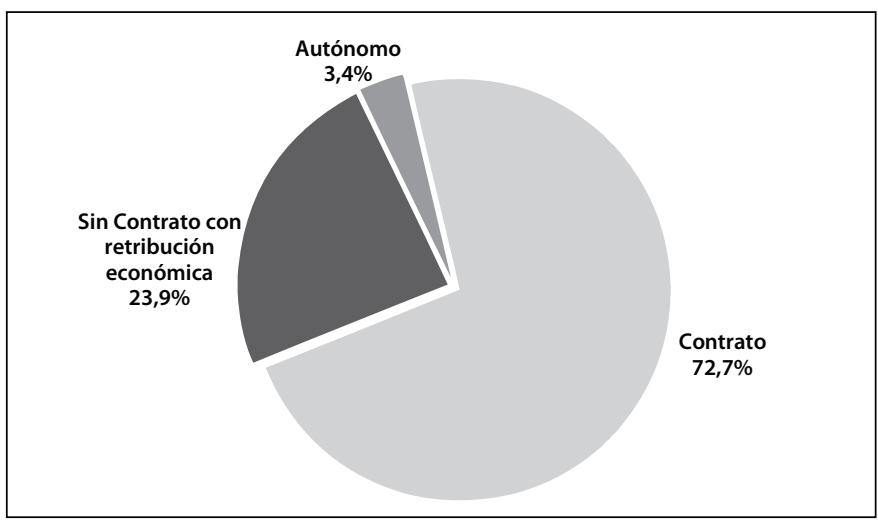

La dedicación de los contratos es a tiempo completo, con un $64 \%$, inferior al $92 \%$ de la población española (Instituto Nacional de Estadística [INE], 2007) y al $86,3 \%$ obtenido por los valencianos (Generalitat Valenciana, 2006). También, Martínez (2007) explica que casi el $75 \%$ de las personas que trabajan en funciones físico-deportivas para los ayuntamientos ha desarrollado anteriormente funciones de actividad física y deporte para otras entidades. En esta línea, un 29,4\% considera su trabajo eventual, un $21,2 \%$ piensa suficiente la cantidad de trabajadores de actividad física en los ayuntamientos y un 81,2\% se plantea continuar trabajando en funciones físicodeportivas durante toda su vida. Para la consecución del puesto de trabajo, ha influido mayoritariamente la biografía personal-curriculum vitae, la entrevista, los contactos y el haber aprobado una oposición (véase Gráfico 3.6). 
Gráfico 3.6. Aspectos que han influido para la consecución del puesto de trabajo actual

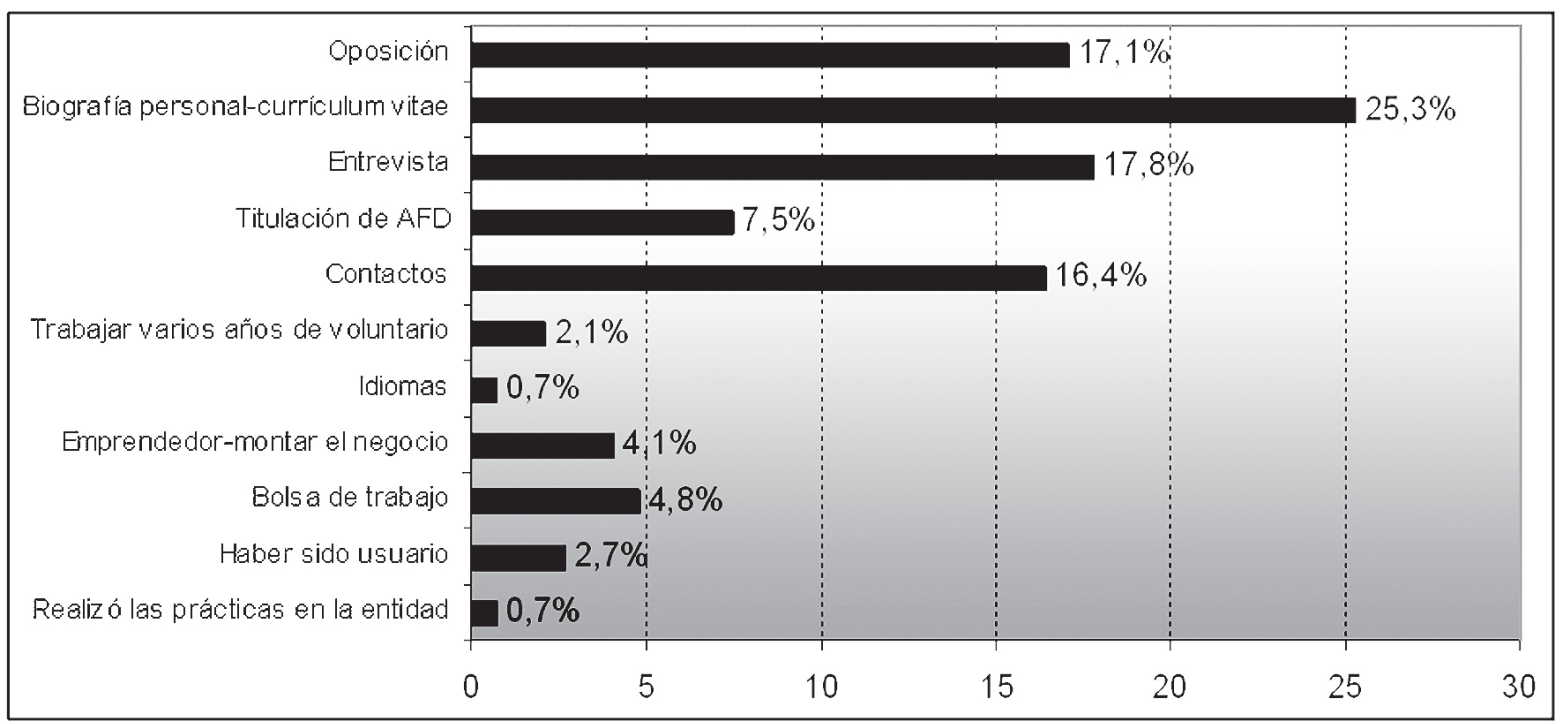

Por último, con respecto a las características formativas, Martínez (2008a, 2008b) establece que un 68,2\% de las personas que tienen funciones físico-deportivas en los ayuntamientos posee alguna de las diferentes titulaciones oficiales de actividad física y deporte. De este porcentaje, un $23,5 \%$ son titulados federativos, un $21,2 \%$ son licenciados en ciencias de la actividad física y del deporte, un $11,8 \%$ son técnicos superiores en animación de actividades físicas y deportivas y un 9,4\% son maestros especialistas en educación física (véase Gráfico 3.7). Este resultado muestra un porcentaje del $31,7 \%$ de personas carentes de titulación deportiva, que están realizando actualmente funciones físico-deportivas para las que no están capacitadas. Al mismo tiempo, en aspectos relativos a la función pública, se describen personas en cargos administrativos y organizativos que han accedido a su puesto de trabajo con otras titulaciones universitarias no relacionadas con el ámbito físico-deportivo (económicas, administración y dirección de empresas, empresariales, etc.), o no acordes con las funciones representadas en el anteproyecto de Ley para la ordenación y regulación del ámbito profesional de la actividad física y del deporte en España (Consejo Superior de Deportes, 2007), en lo que se percibe claramente como un marcado intrusismo en el mercado laboral del licenciado en ciencias de la actividad física y del deporte (Campos, 2005; Campos et al., 2006a, 2006b; Carratalá, Mayorga, Mestre, Montesinos y Rubio, 2004; Jiménez Soto, 2001; Jiménez Soto, Arana y Alcaín, 2005; Martín Acero, 2006a, 2006b, 2007; Martínez et al., 2008a, 2008b; Montalvo, 2007). Por consiguiente, una consecuencia de lo anteriormente comentado es que las propias titulaciones físico-deportivas se solapan y se desarrollan funciones que no se corresponden con la debida titulación de actividad física y deporte.

Gráfico 3.7. Distribución de personas según tipo de titulación de actividad física y deporte y no titulados

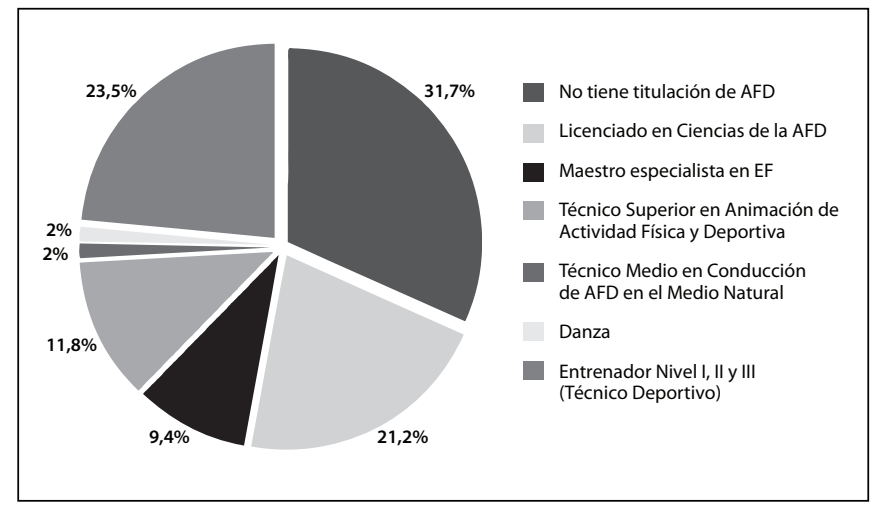

Conclusiones

Se establece el perfil de la persona que trabaja en funciones de actividad física y deporte en los ayuntamientos de la Comunidad Valenciana (España):

Hombre, de 34 años, nacido en la Comunidad Valenciana, que desarrolla varias funciones, principalmente la docencia de actividad física y/o deportes y tareas de organización y coordinación, que efectúa otras labores sin relación con el deporte y que ha trabajado con anterioridad en otras funciones físico-deportivas.

No desea trabajar en funciones diferentes a las actuales y desarrolla sus funciones con diversas poblaciones en instalaciones multideportivas de todas las provincias de la Comunidad Valenciana (España). 
Situación de los recursos humanos de actividad física y deporte en los ayuntamientos de la

comunidad valenciana (España). Gustavo Martínez Serrano, Antonio Campos Izquierdo, Carlos

Pablo Abella y Juan Antonio Mestre Sancho

El contrato que tiene es temporal por obra y servicio o indefinido del tipo funcionario con dedicación a tiempo completo. Lleva trabajando en el ayuntamiento más de 4 años, anteriormente lo hizo entre dos y cuatro entidades; no considera su trabajo eventual, desea trabajar toda su vida en funciones de actividad física y deporte. Considera necesario más personal físicodeportivo, consiguió su trabajo mediante su biografía personal, curriculum vitae, oposición o contactos, y tiene titulación de actividad física y deporte de licenciado o entrenador.

\section{Futuras líneas de investigación}

- Desarrollar la investigación de forma sistemática para establecer una estadística que detalle los cambios que se producen en la Comunidad Autónoma Valenciana a lo largo del tiempo.

- Efectuar un estudio evolutivo de los diferentes titulados en actividad física y deporte ocupados en los ayuntamientos.

- Realizar un estudio en el ámbito estatal, europeo o de otros continentes que sirva para describir y comparar el mercado laboral y profesional de la actividad física y del deporte en otras regiones, provincias y países.

\section{Referencias}

Campos, A. (2005). Situación profesional de las personas que trabajan en funciones de actividad física y deporte en la Comunidad Autónoma Valenciana (2004). Tesis de doctorado para optar al título de Doctor en Ciencias de la Actividad Física y del Deporte, Departamento de Educación Física y Deportiva, Facultad de Ciencias de la Actividad Física y del Deporte de Valencia, Universidad de Valencia, España.

Campos, A., Pablos, C. y Mestre, J.A. (2006a). La estructura y gestión del mercado laboral y profesional de la actividad física y el deporte. Sevilla: Wanceulen.

Campos, A., Pablos, C. y Mestre, J.A. (2006b). Los titulados de la actividad física y del deporte. Sevilla: Wanceulen.

Carratalá, V., Mayorga, J., Mestre, J.A., Montesinos, J.M. y Rubio, S. (2004). Estudio del mercado laboral y de las competencias profesionales del titulado. En F. Del Villar (Coord.), Propuesta de grado en Ciencias de la Actividad Física y el Deporte. Cáceres: Universidad de Extremadura.

Consejo Superior de Deportes. (2007). Borrador del anteproyecto de Ley para la ordenación del ejercicio profesional de la actividad física y el deporte. Madrid: Ministerio de Educación y Ciencia.

García Ferrando, M. (2006). Posmodernidad y deporte: en- tre la individualización y la masificación. Encuesta sobre hábitos deportivos de los españoles 2005. Madrid: CSD.

García Ferrando, M., Mestre, J.A. y Llopis, R. (2007). Los valencianos y el deporte. Valencia: Ajuntament de València.

Generalitat Valenciana. (2006). Anuari estadístic Comunitat Valenciana. Valencia: Arts Gràfiques Soler.

Instituto Valenciano de Estadística. (2006a). Cifras de población referidas al 01/01/2005. Población por municipios y sexo. Manuscrito no publicado.

Instituto Valenciano de Estadística. (2006b). La Comunitat Valenciana en xifres 2006. Valencia: Generalitat Valenciana.

Instituto Nacional de Estadística (2007). Encuesta de población activa. Cuarto trimestre de 2006. Madrid: Instituto Nacional de Estadística.

Jiménez Soto, I. (2001). El ejercicio profesional de las titulaciones del deporte. Barcelona: Bosch.

Jiménez Soto, I., Arana, E. y Alcaín, E. (2005). El derecho deportivo en España, 1975-2005. Sevilla: Consejería de Turismo, Comercio y Deporte.

Martín Acero, R. (Coord.) (2006a). Borrador no 2 del informe de la conferencia española de institutos y facultades de ciencias de la actividad física y del deporte sobre la Ley de ordenamiento y regulación profesional. Manuscrito no publicado, Consejo Superior de Deportes, Madrid, España.

Martín Acero, R. (Coord.) (2006b). Borrador no 3 del informe de la conferencia española de institutos y facultades de ciencias de la actividad física y del deporte sobre la Ley de ordenamiento y regulación profesional. Manuscrito no publicado, Consejo Superior de Deportes, Madrid, España.

Martín Acero, R. (2007). La colaboración de la conferencia española de institutos y facultades de ciencias de la actividad física y del deporte en la propuesta de ley de ordenamiento del ejercicio profesional. Revista de comunicación intercolegial. Consejo General de Ilustres Colegios Oficiales de Licenciados en Educación Física y en Ciencias de la Actividad Física y del Deporte, 2, 2021.

Martínez, G. (2007). Los recursos humanos de la actividad física y del deporte en la Comunidad Autónoma Valenciana. Tesis de doctorado para optar al título de Doctor en Ciencias de la Actividad Física y del deporte, Departamento de Educación Física y Deportiva, Facultad de Ciencias de la Actividad Física y del Deporte de Valencia, Universidad de Valencia, España.

Martínez, G. (2008a). Las características formativas de los recursos humanos de la actividad física y del deporte en la Comunidad Valenciana. Comunicación presentada en el V Congreso de la Asociación Española de Ciencias del Deporte, Universidad de León, León, España.

Martínez, G. (2008b). Social and Demographic Characteristics of Physical Activity and Sport Human Resources 
in Spain. Comunicación presentada en la 5th International Scientific Conference on Kinesiology, Universidad de Zagreb, Zagreb, Croacia.

Martínez, G. (2009). Los recursos humanos de la actividad física y del deporte y sus características laborales. Comunicación presentada en el X Congreso Aeisad deporte, salud y medio ambiente para una sociedad sostenible, Palacio de exposiciones y congresos, Córdoba, España.

Martínez, G., Campos, A., Pablos, C. y Mestre, J.A. (2008a). Los recursos humanos de la actividad física y del deporte: funciones y características sociodemográficas, laborales y formativas. Valencia: Tirant lo Blanch.

Martínez, G., Campos, A., Pablos, C. y Mestre, J.A. (2008b). Las entidades de la actividad física y del deporte: estado actual y perfiles. Valencia: Tirant lo Blanch.

Montalvo, A. (2007). Proyecto de ley para la regulación del ámbito profesional de la actividad física y el deporte. Comunicación presentada en el III Congreso Gestión del Deporte, Universitat Jaume I, Castellón, España. 